\title{
Note About Unstable M3-brane Action
}

\author{
J. Klusoň \\ Department of Theoretical Physics and Astrophysics \\ Faculty of Science, Masaryk University \\ Kotlárská 2, 611 37, Brno \\ Czech Republic \\ E-mail: klu@physics.muni.cz
}

\begin{abstract}
We construct an action for unstable M3-brane in M-theory. We argue that in order to find M3-brane action that upon direct dimensional reduction leads to non-BPS D3-brane action in type IIA theory we have to presume that the background possesses Killing isometry and that this isometry has to be gauged on the world-volume of M3brane. Then we construct singular tachyon kink on world-volume of M3-brane and we show that the dynamics of resulting topological defect is governed by an action that upon direct dimensional reduction leads to D2-brane in type IIA theory and that is equivalent to standard M2-brane action in flat background.
\end{abstract}

KEYWORDs: M-theory. 


\section{Contents}

1. Introduction

2. Naive Form of Unstable M3-brane Action 2

3. Dimensional Reduction 5

4. Proposal for Non-BPS M3-brane 8

5. Double Dimensional Reduction 9

6. Tachyon Kink on M3-Brane 12

7. Gauged M2-Brane Action 15

8. Conclusion 16

\section{Introduction}

Study of unstable systems is an important direction in string theory research, for review and extensive list of references, see [1, 2]. In the context of two superstring theories, type IIA and type IIB, we have two unstable systems: The first one is system that consists Dp-brane and anti-Dp-brane pair with $p$ is odd(even) in type IIA(type IIB) theory. The second one is a single unstable Dp-brane where now $p$ is even(odd) in type IIA(type IIB) theory.

These systems have rich and interesting dynamics. In particular, it is well known that the tachyon condensation on these objects leads to an emergence either stable Dp-branes or fundamental strings. Further, it is remarkable fact that many these phenomena can be described in the language of tachyon effective action ${ }^{1}$.

On the other hand it is well known that all stable D-branes, fundamental strings and other extended objects in string theories arise through dimensional reductions from M2 and M5-branes in M-theory ${ }^{2}$. Then it is natural to ask the question whether it is possible to formulate tachyon effective actions for M-theory unstable objects that could be interpreted as pre-images of unstable D-branes in super string theories. An existence of such objects was predicted in very interesting papers [55] and [61, 62]. We will not try to construct

\footnotetext{
${ }^{1}$ For some works considering effective action description of an unstable systems, see [3, 4, 月, 6, 7, 9, 10 , $11,12,13,14,15,16,17,18,19,24,21,22,23,24,25,26,27,28,29,51,50,49,48,47,46,45,44,43,42$, $41,40,39,38,37,36,35,34,33,32,31$.

${ }^{2}$ For review and extensive list of earlier papers, see [52].
} 
actions for all unstable systems discussed in this paper. We rather restrict ourselves to the case of an unstable M3-brane that can be directly related to M2-brane. In fact, it was argued in [55] that the main property of this M3-brane is that the tachyon kink solution on the world-volume of M3-brane should lead to an emergence of M2-brane. Then motivated by success of the effective action description of the tachyon kink configuration [8, 9, 10] we try to formulate M3-brane effective action that through the tachyon kink solution leads to an action for stable M2-brane ${ }^{3}$.

However we will see that there are some subtleties with construction of an action for such an object. More concretely, we would like to find M3-brane effective action that has two following properties: 1 . The singular tachyon kink condensation leads to an emergence of M2-brane action 2: The dimensional reduction of M3-brane action leads to an unstable D3-brane action in type IIA theory. Unfortunately it turns out that we are not able to obey these two conditions simultaneously. More precisely, in the next section we formulate M3-brane effective action with the property that the singular tachyon kink condensation on its world-volume leads to ordinary M2-brane action. On the other hand, when we perform the dimensional reduction to type IIA theory background we derive the form of the action that is different from the tachyon effective action for unstable D3-brane that is clearly unsatisfactory property of given action.

Then in order to find M3-brane action that reduces to non-BPS D3-brane action in type IIA theory we start our analysis in opposite direction. However it turns out that the construction of given M3-brane action demands an existence of isometry of M-theory supergravity background that has to be gauged on the world-volume of M3-brane action. Then it is clear that singular tachyon condensation on the world-volume of such a M3-brane leads to gauged version of M2-brane action. Then we study properties of this M2-brane action and we find that the direct dimensional reduction leads to D2-brane action. Finally we show that in flat background this action is equivalent to standard M2-brane action.

The organization of this paper is as follows. In next section (2) we introduce the first form of M3-brane action and show that the singular tachyon kink leads to M2-brane action. Then in section (3) we perform direct dimensional reduction in this action. In section (4) we propose alternative form of M3-brane action that upon direct dimensional reduction leads to an unstable D3-brane action. We discuss its double dimensional reduction in section (5) and argue that it leads to unstable NS2-brane. Then in section (6) we also construct tachyon kink solution on its world-volume and we argue that the dynamics of the kink is governed by gauged M2-brane action. We study properties of this action in section (7). Finally in conclusion (8) we outline our results and suggest their possible extension.

\section{Naive Form of Unstable M3-brane Action}

In this section we propose the first form of an unstable M3-brane action with the property that the singular tachyon kink on its world-volume corresponds to a stable M2-brane action

\footnotetext{
${ }^{3}$ We are also motivated by recent progress in the study of effective action for $N$ M2-branes 53 , 54, since we would like to see whether it is possible to find system of unstable $N$ M3-branes that through the kink tachyon condensation leads to the effective action for $N$ M2-branes.
} 
in general background. In fact, with analogy with unstable D3-brane in type IIA theory we propose the action for this unstable M3-brane in the form ${ }^{4}$

$$
S=-\int d^{4} \xi V(T) \sqrt{-\operatorname{det} \mathbf{A}}+\int V(T) d T \wedge C,
$$

where

$$
\mathbf{A}_{\mu \nu}=\hat{g}_{M N} \partial_{\mu} \hat{X}^{M} \partial_{\nu} \hat{X}^{N}+\partial_{\mu} T \partial_{\nu} T
$$

and where $\xi^{\mu}, \mu, \nu=0, \ldots, 3$ label world-volume coordinates of M3-brane. Further, $V(T)$ is the tachyon potential that is even function of $T$ with the property that $V(T= \pm \infty)=0$ and $V(T=0)=\tau_{M 3}$, where $\tau_{M 3}$ is M3-brane tension.

Let us now consider tachyon kink solution on the world-volume of the action (2.1). To do this we closely follow similar analysis performed in case of unstable Dp-branes in general background [8, 9, 10]. We start with the ansatz for world-volume modes

$$
\begin{aligned}
T & =f\left(a\left(\xi^{3}-t\left(\xi^{\alpha}\right)\right)\right), \\
\hat{X}^{M} & =\hat{x}^{M}\left(\xi^{\alpha}\right),
\end{aligned}
$$

where $\xi^{\alpha}, \alpha, \beta=0,1,2$ are coordinates tangential to the world-volume of the kink. The function $f$ introduced in (2.3) satisfies

$$
f(-u)=-f(u), \quad f^{\prime}(u)>0 \quad \forall u, \quad f(\infty)=\infty
$$

but is otherwise an arbitrary function of the argument $u$. $a$ is a constant that we shall take to $\infty$ in the end. In this limit we have $T=\infty$ for $\xi^{3}>0$ and $T=-\infty$ for $\xi^{3}<0$.

The first goal of our analysis is to shown that the action (2.1) evaluated on the ansatz (2.3) leads to the standard M2-brane action in general background

$$
\begin{aligned}
S & =S_{M 2}+S_{W Z}, \\
S_{M 2} & =-T_{M 2} \int d^{3} \xi \sqrt{-\operatorname{det} \mathbf{a}}, \\
S_{W Z} & =-\frac{1}{3 !} T_{M 2} \int d^{3} \xi \epsilon^{\alpha \beta \gamma} \hat{C}_{\alpha \beta \gamma}, \\
\mathbf{a}_{\alpha \beta} & =\partial_{\alpha} \hat{x}^{M} \partial_{\beta} \hat{x}^{N} \hat{g}_{M N}, \quad \hat{C}_{\alpha \beta \gamma}=\hat{C}_{M N K} \partial_{\alpha} \hat{x}^{M} \partial_{\beta} \hat{x}^{N} \partial_{\gamma} \hat{x}^{K}
\end{aligned}
$$

that implies following equations of motion for $\hat{x}^{M}$

$$
\begin{array}{r}
-\frac{1}{2} \frac{\delta \hat{g}_{M N}}{\delta \hat{x}^{K}} \partial_{\alpha} \hat{x}^{M} \partial_{\beta} \hat{x}^{N}\left(\mathbf{a}^{-1}\right)^{\beta \alpha} \sqrt{-\operatorname{det} \mathbf{a}}+ \\
+\partial_{\alpha}\left[\hat{g}_{K M} \partial_{\beta} \hat{x}^{M}\left(\mathbf{a}^{-1}\right)_{S}^{\beta \alpha} \sqrt{-\operatorname{det} \mathbf{a}}\right]+\tilde{J}_{K}=0,
\end{array}
$$

\footnotetext{
${ }^{4}$ We use following [56] conventions for hats. Hats on target space fields indicate they are 11-dimensional. Absence of hats indicates they are 10-dimensional. Further, capital $M, N, \ldots$ label 11-dimensional indices, $m, n$ label 10-dimensional indices.
} 
where $\tilde{J}_{K}=\frac{\delta}{\delta \hat{x}^{K}} S_{W Z}$ and where for letter purposes we introduced the symmetric and antisymmetric form of the matrix $\left(\mathbf{a}^{-1}\right)$

$$
\left(\mathbf{a}^{-1}\right)_{S}^{\alpha \beta}=\frac{1}{2}\left(\left(\mathbf{a}^{-1}\right)^{\alpha \beta}+\left(\mathbf{a}^{-1}\right)^{\beta \alpha}\right), \quad\left(\mathbf{a}^{-1}\right)_{A}^{\alpha \beta}=\frac{1}{2}\left(\left(\mathbf{a}^{-1}\right)^{\alpha \beta}-\left(\mathbf{a}^{-1}\right)^{\beta \alpha}\right) .
$$

As the first step we insert the ansatz (2.3) to the matrix $\mathbf{A}$ and we obtain

$$
\begin{aligned}
& \mathbf{A}_{\alpha \beta}=\partial_{\alpha} \hat{x}^{M} \partial_{\beta} \hat{x}^{N} \hat{g}_{M N}+a^{2} f^{\prime 2} \partial_{\alpha} t \partial_{\beta} t, \\
& \mathbf{A}_{\alpha 3}=-a^{2} f^{\prime 2} \partial_{\alpha} t, \quad \mathbf{A}_{3 \beta}=-a^{2} f^{\prime 2} \partial_{\beta} t, \quad \mathbf{A}_{33}=a^{2} f^{\prime 2}
\end{aligned}
$$

and consequently

$$
\operatorname{det} \mathbf{A}=a^{2} f^{\prime 2} \operatorname{det} \mathbf{a}_{\alpha \beta}
$$

and

$$
\begin{aligned}
& \left(\mathbf{A}^{-1}\right)^{33}=\frac{1}{a^{2} f^{\prime 2}}+\partial_{\alpha} t\left(\mathbf{a}^{-1}\right)^{\alpha \beta} \partial_{\beta} t, \quad\left(\mathbf{A}^{-1}\right)^{\alpha 3}=\left(\mathbf{a}^{-1}\right)^{\alpha \gamma} \partial_{\gamma} t \\
& \left(\mathbf{A}^{-1}\right)^{3 \beta}=\partial_{\gamma} t\left(\mathbf{a}^{-1}\right)^{\gamma \beta}, \quad\left(\mathbf{A}^{-1}\right)^{\alpha \beta}=\left(\mathbf{a}^{-1}\right)^{\alpha \beta} .
\end{aligned}
$$

Then with the help of (2.9) we obtain that the action (2.1) evaluated on the ansatz (2.3) reduces into action (2.5) in the limit $a \rightarrow \infty$

$$
\begin{aligned}
S_{M 3} & =-\int d \xi^{3} d^{3} \xi a f^{\prime} V \sqrt{-\operatorname{det} \mathbf{a}}+\frac{1}{3 !} \int d \xi^{3} a f^{\prime} V d^{3} \xi \epsilon^{\alpha \beta \gamma} \hat{C}_{\alpha \beta \gamma}= \\
& =-T_{M 2} \int d^{3} \xi \sqrt{-\operatorname{det} \mathbf{a}}+T_{M 2} \int \hat{C}
\end{aligned}
$$

where we make standard presumption that

$$
T_{M 2}=\int d x a f^{\prime}(a x) V(f(a x))=\int d u V(u)
$$

As the next step we show that the equations of motion for $T$ and $\hat{X}$ are obeyed for the ansatz (2.3) on condition that the modes $\hat{x}^{M}$ obey the equations of motion (2.6). In fact, it is easy to determine from (2.1) the equation of motion for $T$

$$
-V^{\prime}(T) \sqrt{-\operatorname{det} \mathbf{A}}+\partial_{\mu}\left[V(T) \partial_{\nu} T\left(\mathbf{A}^{-1}\right)_{S}^{\nu \mu} \sqrt{-\operatorname{det} \mathbf{A}}\right]+J_{T}=0
$$

where $J_{T}=\frac{\delta}{\delta T} S_{W Z}$ is the source current derived from varying the Wess-Zumino term. Now using $\mathbf{A}_{\mu \nu}\left(\mathbf{A}^{-1}\right)^{\nu \rho}=\delta_{\mu}^{\rho}$ we obtain

$$
\begin{gathered}
\mathbf{A}_{33}\left(\mathbf{A}^{-1}\right)^{3 \mu}+\mathbf{A}_{3 \alpha}\left(\mathbf{A}^{-1}\right)^{\alpha \mu}=\delta_{3}^{\mu} \Rightarrow \\
\left(\mathbf{A}^{-1}\right)_{S}^{3 \mu}-\partial_{\alpha} t\left(\mathbf{A}^{-1}\right)_{S}^{\alpha \mu}=\frac{1}{a^{2} f^{\prime 2}} \delta_{3}^{\mu}
\end{gathered}
$$


and consequently

$$
\begin{aligned}
& -V^{\prime}(T) \sqrt{-\operatorname{det} \mathbf{A}}+\partial_{\mu}\left[V(T) \partial_{\nu} T\left(\mathbf{A}^{-1}\right)_{S}^{\nu \mu} \sqrt{-\operatorname{det} \mathbf{A}}\right]= \\
& =-V^{\prime}(T) a f^{\prime} \sqrt{-\operatorname{det} \mathbf{a}}+\partial_{\mu}\left[V(T) a^{2} f^{\prime 2}\left(\left(\mathbf{A}^{-1}\right)_{S}^{3 \mu}-\partial_{\alpha} t\left(\mathbf{A}^{-1}\right)_{S}^{\alpha \mu}\right) \sqrt{-\operatorname{det} \mathbf{a}}\right]=0
\end{aligned}
$$

for any $t$. In fact, it can be also easily shown that the tachyon current $J_{T}$ is zero. In other words the tachyon equation of motion is obeyed for any $t$.

Let us now turn to the equations of motion for the scalar modes $\hat{X}^{M}$ that arise from the action (2.1)

$$
\begin{aligned}
& -\frac{1}{2} V \frac{\delta \hat{g}_{M N}}{\delta \hat{X}^{K}} \partial_{\mu} \hat{X}^{M} \partial_{\nu} \hat{X}^{N}\left(\mathbf{A}^{-1}\right)^{\nu \mu} \sqrt{-\operatorname{det} \mathbf{A}}+ \\
& +\partial_{\mu}\left[V \hat{g}_{K M} \partial_{\nu} \hat{X}^{M}\left(\mathbf{A}^{-1}\right)_{S}^{\nu \mu} \sqrt{-\operatorname{det} \mathbf{A}}\right]+J_{K}=0
\end{aligned}
$$

where $J_{K}=\frac{\delta S_{W Z}}{\delta \hat{X}^{K}}$. Inserting the ansatz (2.3) to the equations above we obtain

$$
\begin{aligned}
& -\frac{1}{2} V a f^{\prime} \frac{\delta \hat{g}_{M N}}{\delta \hat{x}^{K}} \partial_{\alpha} \hat{x}^{M} \partial_{\beta} \hat{x}^{N}\left(\mathbf{a}^{-1}\right)^{\alpha \beta} \sqrt{-\operatorname{det} \mathbf{a}}+ \\
& +V a f^{\prime} \partial_{\alpha}\left[\hat{g}_{K M} \partial_{\beta} \hat{x}^{M}\left(\mathbf{a}^{-1}\right)_{S}^{\beta \alpha} \sqrt{-\operatorname{det} \mathbf{a}}\right]+J_{K}= \\
& \left.=a f^{\prime} V[\text { e.o.m. } 6.3)\right]=0
\end{aligned}
$$

using the fact that

$$
\partial_{\alpha}\left[a V f^{\prime}\right]=-\partial_{3}\left[a V f^{\prime}\right] \partial_{\alpha} t
$$

and also

$$
J_{K}=-V(T) \epsilon^{\mu_{1} \ldots \mu_{4}} \partial_{\mu_{1}} T \frac{\delta \hat{C}_{\mu_{2} \ldots \mu_{4}}}{\delta \hat{x}^{K}}=-V(f) a f^{\prime} \epsilon^{\alpha \beta \gamma} \frac{\delta \hat{C}_{\alpha \beta \gamma}}{\delta \hat{x}^{K}}=a f^{\prime} V \tilde{J}_{K}
$$

Let us outline our result. We derived that the dynamics of the tachyon kink is governed by the equations of motion (2.6). In other words we shown that the tachyon kink on the world-volume of M3-brane corresponds to stable M2-brane. This is very nice result and in some sense supports the proposal that (2.1) correctly describes unstable M3-brane. On the other hand as we show below dimensional reduction of this M3-brane does not lead to the correct form of an unstable D3-brane action.

\section{Dimensional Reduction}

In this section we test the properties of M3-brane action (2.1) further when we study the direct dimensional reduction of given action. To do this we will presume that $D=11$ 
background has a $U(1)$ isometry with Killing vector field $\hat{k}^{M}$, such that Lie derivative of all target space fields with respect to $\hat{k}$ vanish:

$$
\mathcal{L}_{\hat{k}} \hat{g}_{M N}=\mathcal{L}_{\hat{k}} \hat{C}_{M N K}=0
$$

In adapted coordinates $\hat{x}^{M}=\left(x^{m}, z\right)$ for which $\hat{k}=\frac{\partial}{\partial z}, \hat{k}^{M}=\delta_{z}^{M}$ we can write the $\mathrm{D}=11$ bosonic fields as

$$
\begin{aligned}
\hat{g}_{m n} & =e^{-\frac{2}{3} \phi} g_{m n}+e^{\frac{4}{3} \phi} C_{m}^{(1)} C_{n}^{(1)}, \\
\hat{g}_{m z} & =-e^{\frac{4}{3} \phi} C_{m}^{(1)}, \quad \hat{g}_{z z}=e^{\frac{4}{3} \phi}, \\
\hat{C}_{m n k}^{(3)} & =C_{m n k}^{(3)}, \quad\left(i_{\hat{k}} \hat{C}\right)_{m n}=B_{m n},
\end{aligned}
$$

where we have $N S \otimes N S$ fields $\left(\phi, g_{m n}, B_{m n}\right)$ and $R \otimes R$ fields $\left(C_{m}^{(1)}, C_{m n k}^{(3)}\right)$. For this ansatz we obtain

$$
\begin{aligned}
\mathbf{A}_{\mu \nu} & =e^{-\frac{2}{3} \phi} g_{m n} \partial_{\mu} X^{m} \partial_{\nu} X^{n}+\partial_{\mu} T \partial_{\nu} T+ \\
& +e^{\frac{4}{3} \phi}\left(\partial_{\mu} X^{m} C_{m}^{(1)}-\partial_{\mu} Z\right)\left(\partial_{\nu} X^{n} C_{n}^{(1)}-\partial_{\nu} Z\right)= \\
& =e^{-\frac{2}{3} \phi}\left[g_{m n} \partial_{\mu} X^{m} \partial_{\nu} X^{n}+e^{\frac{2}{3} \phi} \partial_{\mu} T \partial_{\nu} T+e^{2 \phi} Y_{\mu} Y_{\nu}\right]= \\
& =e^{-\frac{2}{3} \phi} \mathcal{G}_{\mu \rho}\left[\delta_{\nu}^{\rho}+e^{2 \phi}\left(\mathcal{G}^{-1}\right)^{\rho \sigma} Y_{\sigma} Y_{\nu}\right]
\end{aligned}
$$

where we defined

$$
\mathcal{G}_{\mu \nu}=g_{m n} \partial_{\mu} X^{m} \partial_{\nu} X^{n}+e^{\frac{2}{3} \phi} \partial_{\mu} T \partial_{\nu} T, \quad Y_{\mu}=\partial_{\mu} X^{m} C_{m}^{(1)}-\partial_{\mu} Z .
$$

As the next step we use the fact that

$$
\operatorname{det}\left(\mathcal{G}_{\mu \nu}+e^{2 \phi} Y_{\mu} Y_{\nu}\right)=\operatorname{det} \mathcal{G}\left(1+e^{2 \phi}\left(\mathcal{G}^{-1}\right)^{\mu \nu} Y_{\mu} Y_{\nu}\right)
$$

and introduce an auxiliary variable $v$ so that

$$
\begin{gathered}
-\int d^{4} \xi V \sqrt{-\operatorname{det} \mathbf{A}}=\frac{1}{2} \int d^{4} \xi\left(\frac{1}{v} V^{2} \operatorname{det} \mathbf{A}-v\right)= \\
=\frac{1}{2} \int d^{4} \xi\left(\frac{e^{-2 \phi}}{v} V^{2} \operatorname{det} \mathcal{G}+\frac{V^{2}}{v} \operatorname{det} \mathcal{G} Y\left(\mathcal{G}^{-1}\right) Y-v\right) .
\end{gathered}
$$

Further we interpret $Y$ as one form on the world-volume of M3-brane so that $d Z=C^{(1)}-Y$. Then using the fact that $d^{2} Z=0$ we obtain

$$
d\left(C^{(1)}-Y\right)=0
$$


We can consider $Y$ as an independent field when we add to the action an expression

$$
-\int d^{4} \xi F \wedge V(T) d T \wedge\left(C^{(1)}-Y\right)
$$

where $F=d A$ and where $A$ is one-form on the world-volume of M3-brane. Then it is easy to see that the variation of (3.8) with respect to $A$ implies (3.7)

$$
\frac{\delta}{\delta A}\left[-\int d^{4} \xi F \wedge d T \wedge\left(C^{(1)}-Y\right)\right]=-\int d^{4} \xi \delta A d T \wedge d\left(C^{(1)}-Y\right)=0
$$

using the fact that $d(V(T) d T)=V^{\prime} d T \wedge d T=0$. As the next step we use (3.2) together with the definition of $Y$ given in (3.4) to write $\mathrm{WZ}$ term as

$$
\begin{aligned}
S_{W Z} & =-\frac{1}{3 !} \int d^{4} \xi V(T) \epsilon^{\mu_{1} \ldots \mu_{4}} \partial_{\mu_{1}} T \hat{C}_{\mu_{2} \ldots \mu_{4}}= \\
& =-\frac{1}{3 !} \int d^{4} \xi V(T) \epsilon^{\mu_{1} \ldots \mu_{4}} \partial_{\mu_{1}} T\left[C_{m n k}^{(3)} \partial_{\mu_{2}} X^{m} \partial_{\mu_{3}} X^{n} \partial_{\mu_{4}} X^{k}+\right. \\
& \left.+3 B_{m n} \partial_{\mu_{2}} X^{m} \partial_{\mu_{3}} X^{n} C_{\mu_{4}}^{(1)}\right]+ \\
& +\frac{1}{2} \int d^{4} \xi \epsilon^{\mu_{1} \ldots \mu_{4}} V(T) \partial_{\mu_{1}} T B_{m n} \partial_{\mu_{2}} X^{m} \partial_{\mu_{3}} X^{n} Y_{\mu_{4}} .
\end{aligned}
$$

Finally we integrate out $Y$ from the action and we obtain

$$
Y_{\nu}=-\frac{v}{\operatorname{det} \mathcal{G}} \mathcal{G}_{\nu \mu} \epsilon^{\mu \mu_{1} \mu_{2} \mu_{3}}\left(F_{\mu_{1} \mu_{2}}+B_{\mu_{1} \mu_{2}}\right) \partial_{\mu_{3}} T
$$

that we insert back to the action and we obtain

$$
\begin{aligned}
S & =\frac{1}{2} \int d^{4} \xi\left[\frac{e^{-2 \phi} V^{2}}{v} \operatorname{det} \mathcal{G}-\frac{v}{\operatorname{det} \mathcal{G}}\left(\epsilon^{\mu \mu_{2} \mu_{3} \mu_{4}}(B+F)_{\mu_{2} \mu_{3}} \partial_{\mu_{4}} T\right) \mathcal{G}_{\mu \nu}\left(\epsilon^{\nu \nu_{2} \nu_{3} \nu_{4}}(B+F)_{\nu_{2} \nu_{3}} \partial_{\nu_{4}} T\right)\right]- \\
& -\frac{1}{3 !} \int d^{4} \xi \epsilon^{\mu_{1} \ldots \mu_{4}} V \partial_{\mu_{1}} T C_{\mu_{2} \ldots \mu_{4}}^{(3)}-\frac{1}{2} \int d^{4} \xi \epsilon^{\mu_{1} \ldots \mu_{4}} V \partial_{\mu_{1}} T(F+B)_{\mu_{2} \mu_{3}} C_{\mu_{4}}^{(1)} .
\end{aligned}
$$

We can derive an alternative form of the action if we integrate $v$ from (3.12) and hence

$$
\begin{aligned}
S & =-\int d^{4} \xi e^{-\Phi} V \sqrt{-\epsilon^{\mu \mu_{2} \mu_{3} \mu_{4}}(B+F)_{\mu_{2} \mu_{3}} \partial_{\mu_{4}} T \mathcal{G}_{\mu \nu} \epsilon^{\nu \nu_{2} \nu_{3} \nu_{4}}(B+F)_{\nu_{2} \nu_{3}} \partial_{\nu_{4}} T}- \\
& -\frac{1}{3 !} \int d^{4} \xi \epsilon^{\mu_{1} \ldots \mu_{4}} V \partial_{\mu_{1}} T C_{\mu_{2} \ldots \mu_{4}}^{(3)}-\frac{1}{2} \int d^{4} \xi \epsilon^{\mu_{1} \ldots \mu_{4}} V \partial_{\mu_{1}} T(F+B)_{\mu_{2} \mu_{3}} C_{\mu_{4}}^{(1)} .
\end{aligned}
$$

We see that the resulting action is different from the standard unstable D3-brane action and hence it seems to us that the naive form of the action (2.1) is not the correct one that should correspond to M3-brane action. For that reason we suggest an alternative M3-brane action with the main property that its dimensional reduction leads to effective action for an unstable D3-brane. 


\section{Proposal for Non-BPS M3-brane}

In order to construct M3-brane action whose direct dimensional reduction leads to D3-brane action in type IIA theory we proceed in a similar way as in the case of the construction of the massive M-theory KK-monopole action [56]. Following this paper we consider the target space background corresponding to 11-dimensional supergravity with the field content $\left(\hat{g}_{M N}, \hat{C}_{M N K}\right)$ and that has an isometry generated by a Killing vector $\hat{k}^{M}(X)$ such that the Lie derivative of all target space fields with respect to $\hat{k}^{M}$ vanish

$$
\mathcal{L}_{\hat{k}} \hat{C}=\mathcal{L}_{\hat{k}} \hat{g}=0
$$

Then, with analogy with [56] we propose DBI and WZ parts of M3-brane effective action in the form

$$
S_{D B I}=-\int d^{4} \xi V(T)|\hat{k}|^{\frac{1}{2}} \sqrt{-\operatorname{det} \mathbf{A}}
$$

and

$$
S_{W Z}=-\int d^{4} \xi V(T) \epsilon^{\mu_{1} \ldots \mu_{4}} \partial_{\mu_{1}} T \hat{\mathcal{K}}_{\mu_{2} \mu_{3} \mu_{4}}
$$

where

$$
\begin{aligned}
\mathbf{A}_{\mu \nu} & =\hat{g}_{M N} \hat{D}_{\mu} \hat{X}^{M} \hat{D}_{\nu} \hat{X}^{N}+\frac{1}{|\hat{k}|} \hat{\mathcal{F}}_{\mu \nu}+\frac{1}{|\hat{k}|} \partial_{\mu} T \partial_{\nu} T \\
\hat{k}^{2} & =\hat{k}^{M} \hat{k}^{N} \hat{g}_{M N}, \quad \hat{k}^{2}=|\hat{k}|^{2} \\
\hat{\mathcal{F}}_{\mu \nu} & =\partial_{\mu} \hat{b}_{\nu}-\partial_{\nu} \hat{b}_{\mu}+\partial_{\mu} \hat{X}^{M} \partial_{\nu} \hat{X}^{N}\left(i_{\hat{k}} \hat{C}\right)_{M N} \\
\hat{D}_{\mu} X^{M} & =\partial_{\mu} \hat{X}^{M}-\hat{A}_{\mu} \hat{k}^{M}, \quad \hat{A}_{\mu}=\frac{1}{\mid \hat{k}^{2}} \partial_{\mu} \hat{X}^{M} \hat{k}_{M} \\
\hat{\mathcal{K}}_{\mu_{2} \mu_{3} \mu_{4}} & =\partial_{\mu_{2}} \hat{\omega}_{\mu_{3} \mu_{4}}^{(2)}-\partial_{\mu_{3}} \hat{\omega}_{\mu_{2} \mu_{4}}^{(2)}+\partial_{\mu_{4}} \hat{\omega}_{\mu_{2} \mu_{3}}^{(2)}+ \\
& +\frac{1}{3 !} \hat{C}_{K M N} \hat{D}_{\mu_{2}} \hat{X}^{K} \hat{D}_{\mu_{3}} \hat{X}^{M} \hat{D}_{\mu_{4}} \hat{X}^{N}+\frac{1}{2 !} \hat{A}_{\mu_{2}}\left(\partial_{\mu_{3}} \hat{b}_{\mu_{4}}-\partial_{\mu_{4}} \hat{b}_{\mu_{3}}\right)
\end{aligned}
$$

and where, following [56] we introduced non-propagating world-volume 2 -form $\hat{\omega}$ that describes tension of M3-brane.

Let us now discuss the gauge symmetries of the actions (4.2) and (4.3). It is easy to see that they are invariant under local isometry transformation labeled by $\hat{\sigma}^{(0)}$

$$
\begin{aligned}
\delta \hat{X}^{M} & =-\hat{\sigma}^{(0)} \hat{k}^{M}, \quad \delta \hat{g}_{M N}=-\hat{\sigma}^{(0)} \partial_{K} \hat{g}_{M N} \hat{k}^{K} \\
\delta \hat{k}^{M} & =-\hat{\sigma}^{(0)} \partial_{K} \hat{k}^{M} \hat{k}^{K}, \quad \delta|\hat{k}|^{2}=0 \\
\delta \hat{k}_{M} & =\hat{\sigma}^{(0)} \partial_{M} \hat{k}^{K} \hat{k}_{K}, \quad \delta \hat{A}_{\mu}=-\partial_{\mu} \hat{\sigma}^{(0)}, \\
\delta \hat{\omega}_{\mu \nu} & =-\frac{1}{2}\left[\partial_{\mu} \hat{\sigma}^{(0)} \hat{b}_{\nu}-\partial_{\nu} \hat{\sigma}^{(0)} \hat{b}_{\mu}\right] \\
\delta \hat{C}_{K L M} & =-\hat{\sigma}^{(0)} \hat{k}^{N} \partial_{N} \hat{C}_{K L M} .
\end{aligned}
$$


Further, the action is invariant under local gauge transformation labeled by $\hat{\rho}^{(0)}$

$$
\delta \hat{b}_{\mu}=\partial_{\mu} \hat{\rho}^{(0)}, \quad \delta \hat{X}^{M}=0, \quad \delta T=0, \quad \delta \hat{\mathcal{F}}_{\mu \nu}=0
$$

Finally the WZ action is also invariant under the gauge transformation of two form $\hat{\omega}_{\mu \nu}$

$$
\delta \hat{\omega}_{\mu \nu}=\partial_{\mu} \hat{\rho}_{\nu}^{(2)}-\partial_{\nu} \hat{\rho}_{\mu}^{(2)} .
$$

Now we show that for the background (3.2) the actions (4.2), (4.3) describe non-BPS D3brane action in type IIA theory. In fact, when we use (3.2) in (4.4) we obtain

$$
\begin{aligned}
\hat{A}_{\mu} & =-C_{m}^{(1)} \partial_{\mu} X^{m}+\partial_{\mu} Z, \quad \hat{D}_{\mu} X^{m}=\partial_{\mu} X^{m}, \quad \hat{D}_{\mu} Z=|\hat{k}|=e^{\frac{2}{3} \phi}, \\
\mathbf{A}_{\mu \nu} & =e^{-\frac{2}{3} \phi}\left[g_{m n} \partial_{\mu} X^{m} \partial_{\nu} X^{n}+F_{\mu \nu}+B_{m n} \partial_{\mu} X^{m} \partial_{\nu} X^{n}+\partial_{\mu} T \partial_{\nu} T\right] .
\end{aligned}
$$

Then if we insert this result into the action $(4.2)$ we obtain

$$
S=-\int d^{4} \xi e^{-\phi} V(T) \sqrt{-\operatorname{det}\left(g_{m n} \partial_{\mu} X^{m} \partial_{\nu} X^{n}+F_{\mu \nu}+B_{m n} \partial_{\mu} X^{m} \partial_{\nu} X^{n}+\partial_{\mu} T \partial_{\nu} T\right)}
$$

that is clearly the correct form of the D3-brane effective action.

As the next step we perform dimensional reduction in case of WZ term (4.3). In fact, using (4.8) we find

$$
\begin{aligned}
S_{W Z} & =\int d^{4} \xi V(T) \epsilon^{\mu_{1} \ldots \mu_{4}} \partial_{\mu_{1}} T\left[\frac{1}{3 !} C_{m n k}^{(3)} \partial_{\mu_{2}} X^{m} \partial_{\mu_{3}} X^{n} \partial_{\mu_{4}} X^{k}-\right. \\
& -\frac{1}{2 !}\left(\partial_{\mu_{2}} b_{\mu_{3}}-\partial_{\mu_{3}} b_{\mu_{2}}+B_{m n} \partial_{\mu_{2}} X^{m} \partial_{\mu_{3}} X^{n}\right) C_{k}^{(1)} \partial_{\mu_{4}} X^{k}+ \\
& \left.+\left(-\partial_{\mu_{2}} \omega_{\mu_{3} \mu_{4}}+\frac{1}{2} \partial_{\mu_{2}} Z\left(\partial_{\mu_{3}} b_{\mu_{4}}-\partial_{\mu_{4}} b_{\mu_{3}}\right)\right)\right]
\end{aligned}
$$

that is again correct form of the Wess-Zumino term for non-BPS D3-brane that now contains additional contribution from non-propagating two form $\hat{c}_{\mu \nu}^{(2)}=-\hat{\omega}_{\mu \nu}^{(2)}+Z \frac{1}{2}\left(\partial_{\mu} B_{\nu}-\right.$ $\left.\partial_{\nu} B_{\mu}\right)$.

In summary, we found the form of unstable M3-brane action that in natural way-upon direct dimensional reduction-leads to correct form of non-BPS D3-brane action. However it will be interesting to study this unstable M3-brane action further. In the next section we implement double dimensional reduction of given action and try to identify resulting object.

\section{Double Dimensional Reduction}

In this section we briefly discuss the double dimensional reduction of an unstable M3-brane, following [56]. For simplicity we restrict ourselves to the analysis of DBI part of the action only however its extension to WZ term is straightforward. 
In order to perform the double dimensional reduction we have to introduce an extra isometry for the background that is generated by a Killing vector $\hat{h}$ and then wrap one direction of the M3-brane, $\xi^{3}$ around this new compact direction

$$
\partial_{\xi} \hat{X}^{M}=\hat{h}^{M}
$$

Generally we have two different isometries: one is given by $\hat{h}$ that is a direction tangent to a world-volume and the other one is given by $\hat{k}$ in direction transverse to the world-volume. Then the action (4.2) will be invariant under both isometries when $\mathcal{L}_{\hat{h}} \hat{k}=0$. In other words we can find a coordinate system adapted coordinates in both isometries:

$$
\hat{h}^{M}=\delta^{M y}, \quad \hat{k}^{M}=\delta^{M z}
$$

In this double dimensional reduction the Killing vector $\hat{k}$ becomes after reduction the Killing vector associated to the isometry of the space transverse to the world-volume of an unstable M3-brane

$$
\hat{k}^{y}=0, \quad \hat{k}^{m}=k^{m} .
$$

We also use the following reduction rules for the background fields

$$
\begin{aligned}
\hat{g}_{m n} & =e^{-\frac{2}{3} \phi} g_{m n}+e^{\frac{4}{3} \phi} C_{m}^{(1)} C_{n}^{(1)} \\
\hat{g}_{m y} & =-e^{\frac{4}{3}} C_{m}^{(1)}, \quad \hat{g}_{y y}=e^{\frac{4}{3} \phi} \\
\hat{C}_{k m n} & =C_{k m n}^{(3)}, \quad \hat{C}_{m n y}=B_{m n}, \\
\left(i_{\hat{k}} \hat{C}\right)_{m y} & =k^{n} \hat{C}_{m y n}=-\hat{C}_{m n y} k^{n}=-B_{m n} k^{n}=-\left(i_{k} B\right)_{m} .
\end{aligned}
$$

For simplicity we restrict ourselves to the background with zero $C_{m}^{(1)}$. Then we obtain

$$
\hat{k}^{2}=e^{-\frac{2}{3} \phi} k^{2}, \quad k^{2}=g_{m n} k^{m} k^{n}
$$

so that

$$
\hat{A}_{\alpha}=\frac{1}{k^{2}} \partial_{\alpha} X^{m} g_{m n} k^{n} \equiv A_{\alpha}, \quad \hat{A}_{3}=0
$$

Further, we have

$$
\begin{aligned}
\hat{b}_{\alpha} & =b_{\alpha}, \quad \hat{b}_{3}=\omega^{(0)}, \\
\hat{D}_{\alpha} \hat{X}^{m} & =\partial_{\alpha} X^{m}-A_{\alpha} k^{m} \equiv D_{\alpha} X^{m}, \quad \hat{D}_{3} \hat{X}^{m}=0, \\
\hat{\mathcal{F}}_{\alpha \beta} & \equiv \mathcal{F}_{\alpha \beta}=\partial_{\alpha} b_{\beta}-\partial_{\beta} b_{\alpha}+\partial_{\alpha} X^{m} \partial_{\beta} X^{n} C_{m n p}^{(3)} k^{p} \\
\hat{\mathcal{F}}_{\alpha 3} & =-\hat{\mathcal{F}}_{3 \alpha}=\partial_{\alpha} \omega^{(0)}-\partial_{\alpha} X^{m} B_{m n} k^{n} \equiv \mathcal{K}_{\alpha}^{(1)}, \\
\hat{D}_{\alpha} Y & =0, \quad \hat{D}_{3} Y=1 .
\end{aligned}
$$


Then we have

$$
\begin{aligned}
& \mathbf{A}_{\alpha \beta}=e^{-\frac{2}{3} \phi}\left(D_{\alpha} X^{m} D_{\beta} X^{n} g_{m n}+\frac{e^{\phi}}{|k|} \mathcal{F}_{\alpha \beta}+\frac{e^{\phi}}{|k|} \partial_{\alpha} T \partial_{\beta} T\right) \\
& \mathbf{A}_{33}=e^{\frac{4}{3} \phi}, \quad \mathbf{A}_{\alpha 3}=-\mathbf{A}_{3 \alpha}=\frac{e^{\frac{1}{3} \phi}}{|k|} \mathcal{K}_{\alpha}^{(1)}
\end{aligned}
$$

and hence

$$
\begin{aligned}
\operatorname{det} \mathbf{A}_{\mu \nu} & =\operatorname{det}\left(\mathbf{A}_{\alpha \beta}-\mathbf{A}_{\alpha 3}\left(\mathbf{A}^{-1}\right)^{33} \mathbf{A}_{3 \beta}\right) \mathbf{A}_{33}= \\
& =e^{-\frac{2}{3} \phi} \operatorname{det}\left(D_{\alpha} X^{m} D_{\beta} X^{m} g_{m n}+\frac{e^{\phi}}{|k|} \mathcal{F}_{\alpha \beta}+\frac{e^{\phi}}{|k|} \partial_{\alpha} T \partial_{\beta} T+\mathcal{K}_{\alpha}^{(1)} \mathcal{K}_{\beta}^{(1)}\right)
\end{aligned}
$$

so that DBI part of the action takes the form

$$
S=-\int d^{3} \xi V^{\prime}(T) e^{-\frac{1}{2} \phi}|k|^{1 / 2} \sqrt{-\operatorname{det}\left(D_{\alpha} X^{m} D_{\beta} X^{m} g_{m n}+\frac{e^{\phi}}{|k|} \mathcal{F}_{\alpha \beta}+\frac{e^{\phi}}{|k|} \partial_{\alpha} T \partial_{\beta} T+\mathcal{K}_{\alpha}^{(1)} \mathcal{K}_{\beta}^{(1)}\right)}
$$

where

$$
V^{\prime}(T)=\int d \xi^{3} V(T)
$$

It is natural to interpret the double dimensional reduction of an unstable M3-brane as an unstable three-dimensional object in type IIA theory whose existence can be deduced as follows. type IIB theory contains non-BPS D2-brane with tension $\tau_{D 2}=\sqrt{2} \frac{1}{g_{s} l_{s}^{3}}$. Under S-duality rules given as

$$
g_{s}^{\prime}=\frac{1}{g_{s}}, \quad l_{s}^{\prime}=g_{s}^{1 / 2} l_{s}
$$

D2-brane tension transforms as

$$
\tau_{D 2} \rightarrow \frac{\sqrt{2}}{l_{s}^{\prime 3} g_{s}^{\prime 1 / 2}}=\tau_{N S 2}
$$

where the resulting object can be interpreted as unstable NS2-brane.

Let us now perform T-duality between type IIA and type IIB theories with T-duality rules for $g_{s}^{\prime}, l_{s}^{\prime}, R^{\prime}$

$$
\tilde{g}_{s}=\frac{g_{s}^{\prime} l_{s}^{\prime}}{R^{\prime}}, \quad \tilde{l}_{s}=l_{s}^{\prime}, \quad \tilde{R}=\frac{l_{s}^{\prime 2}}{R^{\prime}},
$$

where symbols with tilde correspond to type IIA theory while symbols with bar correspond to type IIB theory. Further we presume that T-duality circle is transverse to the worldvolume of type IIA NS2-brane so that its tension transforms as

$$
\tau_{N S 2} \rightarrow \frac{1}{\tilde{l}_{s}^{3} \tilde{g}_{s}^{1 / 2}} \frac{\tilde{R}^{1 / 2}}{\tilde{l}_{s}^{1 / 2}}
$$


However this agrees with the tension evaluated from the action (5.10) on condition that T-duality circle with radius $\tilde{R}$ coincides with the isometry direction that is transverse to 3-brane in type IIA theory since then $|k|^{1 / 2}=\frac{\tilde{R}^{1 / 2}}{l_{s}^{1 / 2}}$.

In summary, the double dimensional reduction of the action $(4.2)$ leads to the three dimensional object that also naturally arises as a consequence of chains of dualities in type IIA and type IIB theories. We mean that this fact again supports our proposed form of M3-brane action (4.2).

\section{Tachyon Kink on M3-Brane}

We found in the previous section an action for unstable M3-brane action that reduces to non-BPS D3-brane action when we perform direct dimensional reduction. This is very attractive property of given action. However we would like also study the tachyon condensation on given M3-brane and identify the resulting object.

To do this we closely follow analysis presented in section (2) so that we can be brief. We start with the ansatz

$$
\begin{aligned}
T & =f\left(a\left(\xi^{3}-t\left(\xi^{\alpha}\right)\right)\right), \\
\hat{X}^{M} & =\hat{x}^{M}\left(\xi^{\alpha}\right), \quad \hat{b}_{\alpha}=\hat{b}_{\alpha}\left(\xi^{\alpha}\right), \quad \hat{b}_{3}=0,
\end{aligned}
$$

where $\xi^{\alpha}, \alpha, \beta=0,1,2$ are coordinates tangential to the world-volume of the kink. The function $f$ introduced in (6.1) has the same properties as in (2.4).

The first goal of our analysis is to show that the actions (4.2) and (4.3) evaluated on the ansatz (6.1) leads to the following action

$$
\begin{aligned}
S= & S_{D B I}^{M 2 g}+S_{W Z}^{M 2 g}, \\
S^{M 2 g}= & -T_{M 2} \int d^{3} \xi \sqrt{-\operatorname{det} \mathbf{a}}, \quad S^{W Z g}=-T_{M 2} \int d^{3} \xi \epsilon^{\alpha \beta \gamma} \hat{\mathcal{K}}_{\alpha \beta \gamma}, \\
& \mathbf{a}_{\alpha \beta}=\hat{D}_{\alpha} \hat{x}^{M} \hat{D}_{\beta} \hat{x}^{N} \hat{g}_{M N}+\frac{1}{|\hat{k}|} \hat{f}_{\alpha \beta}, \\
\hat{f}_{\alpha \beta}= & \partial_{\alpha} \hat{b}_{\beta}-\partial_{\beta} \hat{b}_{\alpha}+\partial_{\alpha} \hat{x}^{M} \partial_{\beta} \hat{x}^{N}\left(i_{\hat{k}} \hat{C}\right)_{M N}, \\
\hat{\mathcal{K}}_{\alpha \beta \gamma}= & \partial_{\alpha} \hat{\omega}_{\beta \gamma}^{(2)}-\partial_{\beta} \hat{\omega}_{\alpha \gamma}^{(2)}+\partial_{\gamma} \hat{\omega}_{\alpha \beta}^{(2)}+ \\
+ & \frac{1}{3 !} \hat{C}_{K M N}^{(3)} \hat{D}_{\alpha} \hat{x}^{K} \hat{D}_{\beta} \hat{x}^{M} \hat{D}_{\gamma} \hat{x}^{N}+\frac{1}{2 !} \hat{a}_{\alpha}\left(\partial_{\beta} \hat{b}_{\gamma}-\partial_{\gamma} \hat{b}_{\beta}\right) .
\end{aligned}
$$

We will study properties of this action in the next section. Now we determine from (6.2) the equations of motion for $\hat{x}^{M}$

$$
\begin{aligned}
- & \frac{1}{2}\left(\frac{\delta \hat{g}_{M N}}{\delta \hat{x}^{K}} \partial_{\mu} \hat{x}^{M} \partial_{\nu} \hat{x}^{N}-\frac{\delta|\hat{k}|}{\delta \hat{x}^{K}} \frac{1}{|\hat{k}|^{2}} \hat{f}_{\alpha \beta}+\right. \\
& \left.+\frac{1}{|\hat{k}|} \partial_{\alpha} \hat{x}^{M} \partial_{\beta} \hat{x}^{N} \frac{\delta\left(i_{\hat{k}} \hat{C}\right)_{M N}}{\delta \hat{x}^{K}}\right)\left(\mathbf{a}^{-1}\right)^{\beta \alpha} \sqrt{-\operatorname{det} \mathbf{a}}+
\end{aligned}
$$




$$
\begin{aligned}
& +\partial_{\alpha}\left[\hat{g}_{K M} \partial_{\beta} \hat{x}^{M}\left(\mathbf{a}^{-1}\right)_{S}^{\beta \alpha} \sqrt{-\operatorname{det} \mathbf{a}}\right]+ \\
& +\partial_{\alpha}\left[\frac{1}{|\hat{k}|}\left(i_{\hat{k}} \hat{C}\right)_{K M} \partial_{\beta} \hat{x}^{M}\left(\mathbf{a}^{-1}\right)_{S}^{\beta \alpha} \sqrt{-\operatorname{det} \mathbf{a}}\right]+\tilde{J}_{K}=0,
\end{aligned}
$$

where $\tilde{J}_{K}=\frac{\delta}{\delta \hat{x}^{K}} S_{W Z}^{M 2 g}$. Further, the equations of motion for $\hat{b}_{\alpha}$ take the form

$$
\partial_{\alpha}\left[\left(\mathbf{a}^{-1}\right)_{A}^{\beta \alpha} \sqrt{-\operatorname{det} \mathbf{a}}\right]+J_{\alpha}=0, \quad \tilde{J}^{\alpha}=\frac{\delta}{\delta \hat{b}_{\alpha}} S_{W Z}^{M 2 g}=\partial_{\beta}\left[\epsilon^{\alpha \gamma \beta} \hat{a}_{\gamma}\right]
$$

As the first step we insert the ansatz (6.1) to the matrix $\mathbf{A}$ and we get

$$
\begin{aligned}
& \mathbf{A}_{\alpha \beta}=\hat{D}_{\alpha} \hat{x}^{M} \hat{D}_{\beta} \hat{x}^{N} \hat{g}_{M N}+\frac{1}{|\hat{k}|} \hat{f}_{\alpha \beta}+\frac{1}{|\hat{k}|} a^{2} f^{\prime 2} \partial_{\alpha} t \partial_{\beta} t \\
& \mathbf{A}_{\alpha 3}=-\frac{1}{|\hat{k}|} a^{2} f^{\prime 2} \partial_{\alpha} t, \mathbf{A}_{3 \beta}=-\frac{1}{|\hat{k}|} a^{2} f^{\prime 2} \partial_{\beta} t, \mathbf{A}_{33}=\frac{1}{|\hat{k}|} a^{2} f^{\prime 2}
\end{aligned}
$$

Then it is easy to see that

$$
\operatorname{det} \mathbf{A}_{\mu \nu}=\frac{a^{2} f^{\prime 2}}{|\hat{k}|} \operatorname{det} \mathbf{a}_{\alpha \beta}
$$

and also

$$
\begin{aligned}
& \left(\mathbf{A}^{-1}\right)^{33}=\frac{|\hat{k}|}{a^{2} f^{\prime 2}}+\partial_{\alpha} t\left(\mathbf{a}^{-1}\right)^{\alpha \beta} \partial_{\beta} t, \quad\left(\mathbf{A}^{-1}\right)^{\alpha 3}=\left(\mathbf{a}^{-1}\right)^{\alpha \gamma} \partial_{\gamma} t, \\
& \left(\mathbf{A}^{-1}\right)^{3 \beta}=\partial_{\gamma} t\left(\mathbf{a}^{-1}\right)^{\gamma \beta}, \quad\left(\mathbf{A}^{-1}\right)^{\alpha \beta}=\left(\mathbf{a}^{-1}\right)^{\alpha \beta} .
\end{aligned}
$$

Then if we insert (6.6) into (4.2) and take the limit $a \rightarrow \infty$ we obtain

$$
S_{M 3}=-\tau_{M 3} \int d \xi^{3} d^{3} \xi a f^{\prime} V \sqrt{-\operatorname{det} \mathbf{a}}=-T_{M 2} \int d^{3} \xi \sqrt{-\operatorname{det} \mathbf{a}}
$$

where

$$
T_{M 2}=\tau_{M 3} \int d x a f^{\prime}(a x) V(f(a x))=\tau_{M 3} \int d u V(u)
$$

In the same way we can show that when we insert the ansatz (6.1) into (4.3) and take the limit $a \rightarrow \infty$ we derive the WZ part of the action given in (6.2).

As the next step we show that the equations of motion for $T$ and $\hat{X}$ are obeyed for the ansatz (6.1) on condition that the modes $\hat{x}^{M}$ obey the equations of motion (6.3). In fact, it is easy to determine from (4.2) the equation of motion for $T$

$$
-|\hat{k}|^{\frac{1}{2}} V^{\prime}(T) \sqrt{-\operatorname{det} \mathbf{A}}+\frac{1}{2} \partial_{\mu}\left[|\hat{k}|^{\frac{1}{2}} V(T) \frac{1}{|\hat{k}|} \partial_{\nu} T\left(\mathbf{A}^{-1}\right)_{S}^{\nu \mu} \sqrt{-\operatorname{det} \mathbf{A}}\right]+J_{T}=0,
$$


where $J_{T}=\frac{\delta}{\delta T} S_{W Z}$ is the source current derived from varying the WZ term (4.3). Then, following the same analysis as in section (2) it can be easily shown that the tachyon equation of motion are satisfied for any $t$.

Let us now turn to the equations of motion for the scalar modes $\hat{X}^{M}$ that follow from the variation of (4.2) and (4.3)

$$
\begin{aligned}
& -\frac{\delta|\hat{k}|^{\frac{1}{2}}}{\delta \hat{X}^{K}} V \sqrt{-\operatorname{det} \mathbf{A}}-\frac{|\hat{k}|^{\frac{1}{2}}}{2} V\left(\frac{\delta \hat{g}_{M N}}{\delta \hat{X}^{K}} \partial_{\mu} \hat{X}^{M} \partial_{\nu} \hat{X}^{N}-\frac{\delta|\hat{k}|}{\delta \hat{X}^{M}} \frac{1}{|\hat{k}|^{2}}\left(\mathcal{F}_{\alpha \beta}+\partial_{\alpha} T \partial_{\beta} T\right)+\right. \\
& \left.+\frac{1}{|\hat{k}|} \partial_{\mu} \hat{X}^{M} \partial_{\nu} \hat{X}^{N} \frac{\delta\left(i_{\hat{k}} \hat{C}\right)_{M N}}{\delta \hat{X}^{K}}\right)\left(\mathbf{A}^{-1}\right)^{\nu \mu} \sqrt{-\operatorname{det} \mathbf{A}}+ \\
& +\partial_{\mu}\left[|\hat{k}|^{\frac{1}{2}} V \hat{g}_{K M} \partial_{\nu} \hat{X}^{M}\left(\mathbf{A}^{-1}\right)_{S}^{\nu \mu} \sqrt{-\operatorname{det} \mathbf{A}}\right]+ \\
& +\partial_{\mu}\left[|\hat{k}|^{\frac{1}{2}} V \frac{1}{|\hat{k}|}\left(i_{\hat{k}} \hat{C}\right)_{K M} \partial_{\nu} \hat{X}^{M}\left(\mathbf{A}^{-1}\right)_{A}^{\nu \mu} \sqrt{-\operatorname{det} \mathbf{A}}\right]+J_{K}=0
\end{aligned}
$$

where $J_{K}=\frac{\delta S_{W Z}}{\delta \hat{X}^{K}}$. Inserting the ansatz (6.1) to the equation 6.11) we obtain

$$
\begin{aligned}
& -V a f^{\prime} \frac{1}{2}\left(\frac{\delta \hat{g}_{M N}}{\delta \hat{x}^{K}} \partial_{\alpha} \hat{x}^{M} \partial_{\beta} \hat{x}^{N}-\frac{\delta|\hat{k}|}{\delta \hat{x}^{M}} \frac{1}{|\hat{k}|^{2}} \mathcal{F}_{\alpha \beta}+\right. \\
& \left.+\frac{1}{|\hat{k}|} \partial_{\alpha} \hat{x}^{M} \partial_{\beta} \hat{x}^{N} \frac{\delta\left(i_{\hat{k}} \hat{C}\right)_{M N}}{\delta \hat{x}^{K}}\right)\left(\mathbf{a}^{-1}\right)^{\beta \alpha} \sqrt{-\operatorname{det} \mathbf{a}}+ \\
& +\operatorname{Vaf}^{\prime} \partial_{\alpha}\left[\hat{g}_{K M} \partial_{\beta} \hat{x}^{M}\left(\left(\mathbf{a}^{-1}\right)_{S}^{\beta \alpha} \sqrt{-\operatorname{det} \mathbf{a}}\right]+\right. \\
& +a V^{\prime} \partial_{\alpha}\left[\frac{1}{|\hat{k}|}\left(i_{\hat{k}} \hat{C}\right)_{K M} \partial_{\nu} \hat{x}^{M}\left(\mathbf{a}^{-1}\right)_{A}^{\beta \alpha} \sqrt{-\operatorname{det} \mathbf{a}}\right]+J_{K}= \\
& =a f^{\prime} V[\text { eq. of } m . \text { (6.3) }]=0
\end{aligned}
$$

using the fact that

$$
J_{K}=-V(T) \epsilon^{\mu_{1} \ldots \mu_{4}} \partial_{\mu_{1}} T \frac{\delta \hat{\mathcal{K}}_{\mu_{2} \ldots \mu_{4}}}{\delta \hat{x}^{K}}=-V(f) a f^{\prime} \epsilon^{\alpha \beta \gamma} \frac{\delta \hat{\mathcal{K}}_{\alpha \beta \gamma}}{\delta \hat{x}^{K}}=a f^{\prime} V \tilde{J}_{K}
$$

In other words the dynamics of the tachyon kink is governed by the equations of motion (6.3).

As the final step we determine from (4.2) and (4.3) the equation of motion for $\hat{b}_{\mu}$

$$
\partial_{\nu}\left[|\hat{k}|^{\frac{1}{2}} V \frac{1}{|\hat{k}|}\left(\mathbf{A}^{-1}\right)_{A}^{\mu \nu} \sqrt{-\operatorname{det} \mathbf{A}}\right]+J^{\mu}=0,
$$

where $J_{\mu}=\frac{\delta}{\delta \hat{b}_{\mu}} S_{W Z}$. After some manipulations we again find that they are obeyed by the ansatz (6.1) on condition that $\hat{b}_{\alpha}$ obeys 6.4). In summary we have shown that the 
dynamics of the tachyon kink on the world-volume of an unstable M3-brane is governed by the equations of motion that follow from the variation of the action (6.2). Since the form of this action is not well known let us now study its properties.

\section{Gauged M2-Brane Action}

In this section we review the main properties of gauged M2-brane action (6.2). Firstly we show that its dimensional reduction leads to D2-brane action. Further, we demonstrate that the action (2.5) is equivalent to standard M2-brane action in flat background.

We start with the question of dimensional reduction of the action (2.5). We again presume that $D=11$ background has a $U(1)$ isometry with Killing vector field $\hat{k}$ that in

adapted coordinates $\hat{x}^{M}=\left(x^{\mu}, z\right)$ for which $\hat{k}=\frac{\partial}{\partial z}, \hat{k}^{M}=\delta_{Z}^{M}$ so that the background takes the form as in (3.2) so that we obtain

$$
\begin{aligned}
\hat{a}_{\alpha} & =-C_{m}^{(1)} \partial_{\alpha} \hat{x}^{m}+\partial_{\alpha} z, \quad|\hat{k}|=e^{\frac{2}{3} \phi}, \\
\hat{D}_{\alpha} \hat{x}^{m} & =\partial_{\alpha} \hat{x}^{m}, \quad \hat{D}_{\alpha} z=C_{m}^{(1)} \partial_{\alpha} \hat{x}^{m}=C_{\alpha}^{(1)},
\end{aligned}
$$

and hence the matrix a defined in (6.2) takes the form

$$
\mathbf{a}_{\alpha \beta}=e^{-\frac{2}{3} \phi}\left[g_{m n} \partial_{\alpha} x^{m} \partial_{\beta} x^{n}+f_{\alpha \beta}+B_{m n} \partial_{\alpha} x^{m} \partial_{\beta} x^{n}\right] .
$$

Inserting this result into the action (6.2) we obtain

$$
S_{D B I}^{M 2 g}=-T_{M 2} \int d^{3} \xi e^{-\phi} \sqrt{-\operatorname{det}\left(g_{\alpha \beta}+b_{\alpha \beta}+f_{\alpha \beta}\right)}
$$

that is the correct form of DBI action for D2-brane. In the same way we can show that the WZ term given in (6.2) takes the form

$$
\begin{aligned}
S_{W Z}^{M 2 g}= & T_{3} \int d^{3} \xi \epsilon^{\alpha \beta \gamma}\left(\frac{1}{3 !} C_{m n k}^{(3)} \partial_{\alpha} x^{m} \partial_{\beta} x^{n} \partial_{\gamma} x^{k}-\right. \\
& -\frac{1}{2 !}\left(\partial_{\alpha} b_{\beta}-\partial_{\beta} b_{\alpha}+B_{m n} \partial_{\alpha} x^{m} \partial_{\beta} x^{n}\right) C_{k}^{(1)} \partial_{\gamma} x^{k}+ \\
& +\left(-\partial_{\alpha} \omega_{\beta \gamma}+\frac{1}{2} \partial_{\alpha} z\left(\partial_{\beta} b_{\gamma}-\partial_{\gamma} b_{\beta}\right)\right)
\end{aligned}
$$

This can be considered as the Wess-Zumino term for D2-brane when we introduce nonpropagating world-volume two-form $c^{(2)}$ as

$$
c_{\alpha \beta}^{(2)}=-\hat{\omega}_{\alpha \beta}^{(2)}+\frac{z}{2}\left[\partial_{\alpha} b_{\beta}-\partial_{\beta} b_{\alpha}\right] .
$$

Finally we show that the action (2.5) is equivalent to standard M2-brane action in the flat eleven dimensional space-time with $\hat{g}_{M N}=\eta_{M N}, \hat{C}_{M N K}=0$. Using $S O(10)$ rotation 
symmetry of spatial part of target space-time we can take $\hat{k}^{M}=\delta_{10}^{M}$ so that $\hat{A}_{\alpha}=\partial_{\alpha} \hat{X}^{10}$ so that

$$
\hat{D}_{\alpha} \hat{X}^{m}=\partial_{\alpha} \hat{X}^{m}, m=0, \ldots, 9, \quad \hat{D}_{\alpha} \hat{X}^{10}=0 .
$$

Then DBI part of M2-brane action takes the form

$$
\begin{aligned}
S_{D B I}^{M 2 g} & =-T_{M 2} \int d^{3} \xi \sqrt{-\operatorname{det}\left(\partial_{\alpha} \hat{x}^{m} \partial_{\beta} \hat{x}^{n} \eta_{m n}+f_{\alpha \beta}\right)}= \\
& =-T_{M 2} \int d^{3} \xi \sqrt{-\operatorname{det}\left(\partial_{\alpha} \hat{x}^{m} \partial_{\beta} \hat{x}^{n} \eta_{m n}+\frac{1}{T_{M 2}^{2}} B_{\alpha} B_{\beta}\right)}+\frac{1}{2} \int d^{3} \xi \epsilon^{\alpha \beta \gamma} B_{\alpha} f_{\beta \gamma} .
\end{aligned}
$$

Then if we integrate out $b_{\alpha}$ from the equation above we obtain

$$
\partial_{\alpha} \epsilon^{\alpha \beta} B_{\beta}=0
$$

that can be solved as $B_{\alpha}=T_{M 2} \partial_{\alpha} \hat{x}^{10}$. Inserting this result to the action above and performing integration by parts we finally get

$$
S=-T_{M 2} \int d^{3} \xi \sqrt{-\operatorname{det} \partial_{\alpha} \hat{x}^{M} \partial_{\beta} \hat{x}^{N}}
$$

that is standard M2-brane action in flat background.

\section{Conclusion}

Now we summarize our results. The initial goal was to find the action for unstable M3-brane that is related by direct dimensional reduction to an unstable D3-brane action and the singular tachyon kink on its world-volume leads to stable M2-brane action. We argued that it is not possible to obey these two conditions simultaneously. Then we suggested alternative form of M3-brane effective action that however exists on condition that the supergravity background has Killing isometry that is gauged on the world-volume of M3-brane. We also shown that the tachyon kink solution on its world-volume leads to M2-brane action that is equivalent to the standard M2-brane action. This fact suggests that the proposed M3-brane action could be starting point for its non-abelian generalization. It would be also really interesting to perform the same procedure as in paper [60] in this M3-brane action and try to find the relation with recent works [58, 59].

Acknowledgement: This work was supported by the Czech Ministry of Education under Contract No. MSM 0021622409.

\section{References}

[1] A. Sen, "Non-BPS states and branes in string theory," arXiv:hep-th/9904207.

[2] A. Sen, "Tachyon dynamics in open string theory," Int. J. Mod. Phys. A 20 (2005) 5513 [arXiv:hep-th/0410103]. 
[3] M. R. Garousi and E. Hatefi, "On Wess-Zumino terms of Brane-Antibrane systems," Nucl. Phys. B 800 (2008) 502 [arXiv:0710.5875 [hep-th]].

[4] M. R. Garousi, "On the effective action of D-brane-anti-D-brane system," JHEP 0712 (2007) 089 [arXiv:0710.5469 [hep-th]].

[5] I. Cho, T. Kim, Y. Kim and K. Ryu, "Relativistic Dynamics of Multi-BPS D-vortices and Straight BPS D-strings," arXiv:0707.0357 [hep-th].

[6] J. Kluson and K. L. Panigrahi, "On the Universal Tachyon and Geometrical Tachyon," JHEP 0706 (2007) 015 [arXiv:0704.3013 [hep-th]].

[7] J. Kluson, "Non-relativistic non-BPS Dp-brane," Nucl. Phys. B 765 (2007) 185 [arXiv:hep-th/0610073].

[8] A. Sen, "Dirac-Born-Infeld action on the tachyon kink and vortex," Phys. Rev. D 68 (2003) 066008 [arXiv:hep-th/0303057].

[9] J. Kluson, "Tachyon kink on non-BPS Dp-brane in the general background," JHEP 0510 (2005) 076 [arXiv:hep-th/0508239].

[10] J. Kluson, "Note about tachyon kink in nontrivial background," JHEP 0508 (2005) 032 [arXiv:hep-th/0506250].

[11] M. R. Garousi and K. B. Fadafan, "Stable false vacuum," JHEP 0604 (2006) 005 [arXiv:hep-th/0506055].

[12] C. Kim, Y. Kim, H. h. Kwon and O. K. Kwon, "BPS D-branes from an unstable D-brane in a curved background," Phys. Rev. D 72 (2005) 026006 [arXiv:hep-th/0504130].

[13] M. R. Garousi, "D-brane anti-D-brane effective action and brane interaction in open string channel," JHEP 0501 (2005) 029 [arXiv:hep-th/0411222].

[14] R. Banerjee, Y. Kim and O. K. Kwon, "Noncommutative tachyon kinks as D(p-1)-branes from unstable D p-brane," JHEP 0501 (2005) 023 [arXiv:hep-th/0407229].

[15] J. Kluson, "Proposal for the open string tachyon effective action in the linear dilaton background," JHEP 0406 (2004) 021 [arXiv:hep-th/0403124].

[16] H. U. Yee and P. Yi, "Open / closed duality, unstable D-branes, and coarse-grained closed strings," Nucl. Phys. B 686 (2004) 31 [arXiv:hep-th/0402027].

[17] V. Niarchos, "Notes on tachyon effective actions and Veneziano amplitudes," Phys. Rev. D 69 (2004) 106009 [arXiv:hep-th/0401066].

[18] A. Sen, "Moduli space of unstable D-branes on a circle of critical radius," JHEP 0403 (2004) 070 [arXiv:hep-th/0312003].

[19] A. Fotopoulos and A. A. Tseytlin, "On open superstring partition function in inhomogeneous rolling tachyon background," JHEP 0312 (2003) 025 [arXiv:hep-th/0310253].

[20] M. Smedback, "On effective actions for the bosonic tachyon," JHEP 0311 (2003) 067 [arXiv:hep-th/0310138].

[21] C. j. Kim, Y. b. Kim, O. K. Kwon and P. Yi, "Tachyon tube and supertube," JHEP 0309 (2003) 042 [arXiv:hep-th/0307184].

[22] O. K. Kwon and P. Yi, "String fluid, tachyon matter, and domain walls," JHEP 0309 (2003) 003 [arXiv:hep-th/0305229]. 
[23] C. Kim, Y. Kim, O. K. Kwon and C. O. Lee, "Tachyon kinks on unstable Dp-branes," JHEP 0311 (2003) 034 [arXiv:hep-th/0305092].

[24] A. Sen, "Open and closed strings from unstable D-branes," Phys. Rev. D 68 (2003) 106003 [arXiv:hep-th/0305011].

[25] P. Brax, J. Mourad and D. A. Steer, "Tachyon kinks on non BPS D-branes," Phys. Lett. B $\mathbf{5 7 5}$ (2003) 115 [arXiv:hep-th/0304197].

[26] C. j. Kim, Y. b. Kim and C. O. Lee, "Tachyon kinks," JHEP 0305 (2003) 020 [arXiv:hep-th/0304180].

[27] K. Okuyama, "Wess-Zumino term in tachyon effective action," JHEP 0305 (2003) 005 [arXiv:hep-th/0304108].

[28] D. Kutasov and V. Niarchos, "Tachyon effective actions in open string theory," Nucl. Phys. B 666 (2003) 56 [arXiv:hep-th/0304045].

[29] M. R. Garousi, "Off-shell extension of S-matrix elements and tachyonic effective actions," JHEP 0304 (2003) 027 [arXiv:hep-th/0303239].

[30] A. Sen, "Supersymmetric world-volume action for non-BPS D-branes," JHEP 9910, 008 (1999) [arXiv:hep-th/9909062].

[31] M. R. Garousi, "Tachyon couplings on non-BPS D-branes and Dirac-Born-Infeld action," Nucl. Phys. B 584, 284 (2000) [arXiv:hep-th/0003122].

[32] M. R. Garousi, "On-shell S-matrix and tachyonic effective actions," Nucl. Phys. B 647, 117 (2002) [arXiv:hep-th/0209068].

[33] E. A. Bergshoeff, M. de Roo, T. C. de Wit, E. Eyras and S. Panda, "T-duality and actions for non-BPS D-branes," JHEP 0005, 009 (2000) [arXiv:hep-th/0003221].

[34] J. Kluson, "Proposal for non-BPS D-brane action," Phys. Rev. D 62, 126003 (2000) [arXiv:hep-th/0004106].

[35] J. A. Minahan and B. Zwiebach, "Gauge fields and fermions in tachyon effective field theories," JHEP 0102, 034 (2001) [arXiv:hep-th/0011226].

[36] M. Alishahiha, H. Ita and Y. Oz, "On superconnections and the tachyon effective action," Phys. Lett. B 503, 181 (2001) [arXiv:hep-th/0012222].

[37] N. D. Lambert and I. Sachs, "Tachyon dynamics and the effective action approximation," Phys. Rev. D 67, 026005 (2003) [arXiv:hep-th/0208217].

[38] J. A. Minahan, "Rolling the tachyon in super BSFT," JHEP 0207, 030 (2002) [arXiv:hep-th/0205098].

[39] D. Kutasov, M. Marino and G. W. Moore, "Remarks on tachyon condensation in superstring field theory," arXiv:hep-th/0010108.

[40] N. D. Lambert and I. Sachs, "On higher derivative terms in tachyon effective actions," JHEP 0106, 060 (2001) [arXiv:hep-th/0104218].

[41] K. Hashimoto and S. Hirano, "Metamorphosis of tachyon profile in unstable D9-branes," Phys. Rev. D 65, 026006 (2002) [arXiv:hep-th/0102174].

[42] G. Arutyunov, S. Frolov, S. Theisen and A. A. Tseytlin, "Tachyon condensation and universality of DBI action," JHEP 0102, 002 (2001) [arXiv:hep-th/0012080]. 
[43] G. W. Gibbons, K. Hori and P. Yi, "String fluid from unstable D-branes," Nucl. Phys. B 596, 136 (2001) [arXiv:hep-th/0009061].

[44] P. Mukhopadhyay and A. Sen, "Decay of unstable D-branes with electric field," JHEP 0211, 047 (2002) [arXiv:hep-th/0208142].

[45] A. Sen, "Some issues in non-commutative tachyon condensation," JHEP 0011, 035 (2000) [arXiv:hep-th/0009038].

[46] A. Sen, "Fundamental strings in open string theory at the tachyonic vacuum," J. Math. Phys. 42, 2844 (2001) [arXiv:hep-th/0010240].

[47] J. A. Minahan and B. Zwiebach, "Effective tachyon dynamics in superstring theory," JHEP 0103, 038 (2001) [arXiv:hep-th/0009246].

[48] A. Sen, "Rolling tachyon," JHEP 0204, 048 (2002) [arXiv:hep-th/0203211].

[49] A. Sen, “Tachyon matter,” JHEP 0207, 065 (2002) [arXiv:hep-th/0203265].

[50] A. Sen, "Time evolution in open string theory," JHEP 0210, 003 (2002) [arXiv:hep-th/0207105].

[51] P. Yi, "Membranes from five-branes and fundamental strings from Dp branes," Nucl. Phys. B 550, 214 (1999) [arXiv:hep-th/9901159].

[52] P. K. Townsend, "Four lectures on M-theory," arXiv:hep-th/9612121.

[53] J. Bagger and N. Lambert, "Comments On Multiple M2-branes," JHEP 0802 (2008) 105 [arXiv:0712.3738 [hep-th]].

[54] J. Bagger and N. Lambert, "Gauge Symmetry and Supersymmetry of Multiple M2-Branes," Phys. Rev. D 77 (2008) 065008 [arXiv:0711.0955 [hep-th]].

[55] K. A. Intriligator, M. Kleban and J. Kumar, "Comments on unstable branes," JHEP 0102, 023 (2001) [arXiv:hep-th/0101010].

[56] E. Bergshoeff, E. Eyras and Y. Lozano, "The massive Kaluza-Klein monopole," Phys. Lett. B 430 (1998) 77 [arXiv:hep-th/9802199].

[57] E. Bergshoeff, Y. Lozano and T. Ortin, "Massive branes," Nucl. Phys. B 518 (1998) 363 [arXiv:hep-th/9712115].

[58] M. R. Garousi, “A proposal for M2-brane-anti-M2-brane action,” arXiv:0809.0381 [hep-th].

[59] R. Iengo and J. G. Russo, "Non-linear theory for multiple M2 branes," arXiv:0808.2473 [hep-th].

[60] J. Kluson, "D2 to M2 Procedure for D2-Brane DBI Effective Action," arXiv:0807.4054 [hep-th].

[61] L. Houart and Y. Lozano, "Brane descent relations in M-theory," Phys. Lett. B 479 (2000) 299 [arXiv:hep-th/0001170].

[62] L. Houart and Y. Lozano, "S-duality and brane descent relations," JHEP 0003 (2000) 031 [arXiv:hep-th/9911173]. 